\title{
Quorum Sensing Inhibitors as Anti-Pathogenic Drugs in the Fight Against Pseudomonas aeruginosa Infections
}

\section{Ani loana Cotar* \\ National Institute for Research and Development in Microbiology and Immunology, Romania}

Keywords: Anti-pathogenic strategy; Multidrug resistance; $P$. aeruginosa; Quorum-sensing inhibitors; Probiotics; New vegetal compounds with anti-pathogenic activity

The increasing of antibiotic resistance among clinically P. aeruginosa strains determined researchers to find out new alternatives to antibiotic treatment. The newest strategy in therapeutics development is represented by an alternative approach which aims to target functions essential for infection, such as virulence factors required to cause host damage and disease. This approach has several potential advantages including expanding the repertoire of bacterial targets, preserving the host endogenous microbiome, and exerting less selective pressure, which may result in decreased resistance [1]. Most efforts to inhibit the regulation of virulence factors expression have focused on interfering with quorum sensing (QS). Because of the significant role of QS in the regulation of hundreds of virulence factors in $\mathbf{P}$. aeruginosa, significant efforts have been made to discover compounds with inhibitory activity of QS systems, that could stay to the base of an anti-pathogenic strategy, since they will inhibit the coordinated expression of virulence determinants, without interfering with bacterial growth, being less likely to generate resistance [2]. Many studies that have shown that deletion of one or more QS genes result in reduced P. aeruginosa virulence compared with the wild-type $P$. aeruginosa [3].

Many groups of researchers from worldwide had focused to find out the most appropriate quorum sensing inhibitors (QSI) that could be used for the inhibition of pathogenicity and virulence of multidrug resistant $P$. aeruginosa strains. Of the numerous compounds able to inhibit QS a few have been tested in animal models with great success. Unfortunately, these compounds are unsuitable for human use. The halogenated furanones are unstable and the fungal compounds identified so far are mycotoxins [4].

In the last years many studies have been concentrated to finding different plant extracts that could be use as QSI. Thus, plants such carrots, garlic, habanero (chili), and water lily produce compounds that interfere with bacterial QS [4]. In addition the waxy compound, propolis, produced by bees also contains QSI activity. DNA array analysis revealed that the garlic extract targets 34\% of the QS genes in $P$. aeruginosa. Other plants including pea (Pisum sativum) seedlings, crown vetch, soybean, ginseng (Panax ginseng), and tomato have also been found to be able to interfere with bacterial QS [5]. Ginseng could represents a source of QSI, because the results of a study have shown that the extract of ginseng suppressed the production of LasA and LasB proteases, did not inhibit the growth of the bacteria, and downregulated the synthesis of the AHL molecules. Though ginseng is a promising natural synergetic remedy, but it is important to isolate and evaluate the ginseng compounds associated with the anti-QS activity [6]. In the majority of analyzed plant extracts that exhibited QSI activity are necessary studies to isolate and characterise the compounds with inhibitory activity against QS.

A new strategy of virulence attenuation can be based on metabolites produced by probiotic strains. Many studies have shown that some probiotic strains exhibit inhibitory activity on different virulence properties of pathogenic bacteria (adherence to cellular or inert substrate, soluble virulence factors expression) [7,8]. The use of probiotics to prevent and treat a wide variety of community-associated and nosocomial infections has been gaining favourable attention recently. This is in part due to a need to find alternatives to traditional antibiotic therapies [9]. The antimicrobial activities of Lactobacillus strains are well known against enteric pathogens including Escherichia coli, S. typhi, Shigella sp., etc. [10,11]. To date, Lactobacilli species have only been used to treat gastrointestinal and urogenital tract disorders [11]. However, their ability to secrete acids, bacteriocins and other by-products that may neutralise infection caused by pathogens, and the fact that they are considered to be harmless microorganisms that regulate the host's inflammatory and immune responses, mean that lactobacilli may be useful for the treatment of clinical infections in other parts of the body, such as recurrent bladder infections caused by Escherichia coli, vaginosis caused by anaerobic microbes, wound infections caused by Staphylococcus aureus, and others [12-14], In the last years were performed few studies regarding the interference of different probiotic strains from Lactobacillus genus with the pathogenic properties of $P$. aeruginosa, and aimed also to to define some of the mechanisms involved $[15,16]$. The results obtained in these studies have shown that the compounds secreted by probiotics exhibited inhibitory activity on growth, cytotoxicity and biofilm formation of several $P$. aeruginosa strains [11]. Also, the results of one study suggest that $L$. fermentum-secreted compounds may also have applications in other types of clinical infections than for the local treatment of $P$. aeruginosa burn infections. Further study on the mechanism of these activities and identification of the antimicrobial compound(s) secreted by $L$. fermentum will help to design more effective antimicrobials and therapeutic approaches in treating hospital-acquired infections [16].

In our studies we have shown that soluble molecules accumulated in the probiotic supernatant of Lactobacillus paracasei subsp. paracasei CMGB 18 culture filtrate (PCF), isolated from newborn faeces, are exhibiting inhibitory properties on $P$. aeruginosa strains growth, adherence to the inert substratum [17] and on QS genes expression level [7]. We have further aimed to detect and quantify the soluble molecules accumulated in PCF, and to investigate their inhibitory activity on $P$. aeruginosa $\mathrm{QS}$ genes expression level. In a recent study we have shown by real-time RT-qPCR that the sub-inhibitory concentrations of organic acids (acetic acid and lactic acid) secreted by the same probiotic strain

*Corresponding author: Ani loana Cotar, Laboratory for Vector-Borne Infections, National Institute for Research and Development in Microbiology and Immunology, Romania, Tel: + 40213609 243; E-mail: aniioana@yahoo.com

Received May 06, 2013; Accepted May 08, 2013; Published May 10, 2013

Citation: Cotar Al (2013) Quorum Sensing Inhibitors as Anti-Pathogenic Drugs in the Fight Against Pseudomonas aeruginosa Infections. Clin Microbial 2: e111. doi:10.4172/2327-5073.1000e111

Copyright: () 2013 Cotar Al. This is an open-access article distributed under the terms of the Creative Commons Attribution License, which permits unrestricted use, distribution, and reproduction in any medium, provided the original author and source are credited. 
Citation: Cotar Al (2013) Quorum Sensing Inhibitors as Anti-Pathogenic Drugs in the Fight Against Pseudomonas aeruginosa Infections. Clin Microbial 2: e111. doi:10.4172/2327-5073.1000e111

decreased the QS genes expression in P. aeruginosa multidrug resistant strains grown in the presence of these acids comparatively with with the expression level in control strains grown in normal conditions [1]. In conclusion these metabolites (soluble molecules) secreted by probiotic strain have inhibitory activity on the QS genes expression, and could represent QSI.

At present the researchers from worldwide are interested to find out the most appropriate QSI that could be used for the inhibition of virulence of multidrug resistant $P$. aeruginosa strains. Whether QSIs offer new hope in the continuing battle against multi-antibioticresistant bacteria is not yet fully apparent but the results certainly appear promising. Not only is expression of many virulence factors down regulated by QSI compounds but the inhibitors also render biofilm bacteria more susceptible to conventional anti-microbial treatments. In spite of the enormous number of QSIs investigated until now, no one of these compounds was investigated in clinical trials in order to be used in treatment of $P$. aeruginosa infections.

\section{References}

1. Cotar Al, Saviuc C, Nita RA, Bezirtzoglou E, Chifiriuc MC (2013)Anti-pathogenic Strategies for Fighting Pseudomonas aeruginosa Infections- probiotic Soluble Compounds as Inhibitors Of Quorum Sensing Genes Expression. Current Organic Chemistry 17: 155-161.

2. Smith RS, Iglewski BH (2003) P. aeruginosa quorum-sensing systems and virulence. Curr Opin Microbiol 6: 56-60.

3. Rasmussen TB, Givskov M (2006) Quorum-sensing inhibitors as antipathogenic drugs. Int J Med Microbiol 296: 149-161.

4. Rasmussen TB, Skindersoe ME, Bjarnsholt T, Phipps RK, Christensen KB et al. (2005) Identity and effects of quorum-sensing inhibitors produced by Penicillium species. Microbiology 151: 1325-1340.

5. Persson T, Hansen TH, Rasmussen TB, Skindersø ME, Givskov M, et al. (2005) Rational design and synthesis of new quorum-sensing inhibitors derived from acylated homoserine lactones and natural products from garlic. Org Biomol Chem 3: 253-262.

6. Song Z, Kong KF, Wu H, Maricic N, Ramalingam B, et al. (2010) Panax ginseng has anti-infective activity against opportunistic pathogen Pseudomonas aeruginosa by inhibiting quorum sensing, a bacterial communication process critical for establishing infection. Phytomedicine 17: 1040-1046.

7. Cotar Al, Chifiriuc MC, Dinu S, Pelinescu D, Banu O, et al. (2010) Quantitative real-time PCR study of the influence of probiotic culture soluble fraction on the expression of Pseudomonas aeruginosa quorum sensing genes. Roum Arch Microbiol Immunol 69: 213-223.

8. Iordache F, lordache C, Chifiriuc MC, Bleotu C, Smarandache D, et al. (2008) Antimicrobial and immunomodulatory activity of some probiotic fractions with potential clinical application. Archiva Zootechnica 11: 41-51.

9. Viswanathan VK, Hodges K, Hecht G (2009) Enteric infection meets intestinal function: how bacterial pathogens cause diarrhoea. Nat Rev Microbiol 7: 110119

10. Servin AL (2004) Antagonistic activities of lactobacilli and bifidobacteria against microbial pathogens. FEMS Microbiol Rev 28: 405-440.

11. Varma P, Dinesh KR, Menon KK, Biswas R (2010) Lactobacillus fermentum isolated from human colonic mucosal biopsy inhibits the growth and adhesion of enteric and foodborne pathogens. J Food Sci 75: M546-M551.

12. Hessle C, Andersson B, Wold AE (2000) Gram-positive bacteria are potent inducers of monocytic interleukin-12 (IL-12) while gram-negative bacteria preferentially stimulate IL-10 production. Infect Immun 68: 3581-3586.

13. Reid G (2001) Probiotic agents to protect the urogenital tract against infection. Am J Clin Nutr 73: 437S-443S.

14. Gan BS, Kim J, Reid G, Cadieux P, Howard JC (2002) Lactobacillus fermentum RC-14 inhibits Staphylococcus aureus infection of surgical implants in rats. J Infect Dis 185: 1369-1372.

15. Valdéz JC, Peral MC, Rachid M, Santana M, Perdigón G (2005) Interference of Lactobacillus plantarum with Pseudomonas aeruginosa in vitro and in infected burns: the potential use of probiotics in wound treatment. Clin Microbiol Infect 11: $472-479$.

16. Varma P, Nisha N, Dinesh KR, Kumar AV, Biswas R (2011) Anti-infective properties of Lactobacillus fermentum against Staphylococcus aureus and Pseudomonas aeruginosa. J Mol Microbiol Biotechnol 20: 137-143.

17. Chifiriuc MC, Ditu ML, Banu O, Bleotu C, Dracea O, et al. (2009) Subinhibitory concentrations of phenyl lactic acid interfere with the expression of virulence factors in Staphylococcus aureus and Pseudomonas aeruginosa clinical strains. Roum Arch Microbiol Immunol 68: 27-33. 